\title{
EMBRIOLOGÍA DE LAS ESTRUCTURAS REPRODUCTORAS MASCUlinas DEL GÉNERO PINGUiCUla L. (LENTIBULARIACEAE)
}

\author{
Silvia Espinosa-Matías ${ }^{1,3}$, Sergio Zamudio² y Judith MárqueZ-GuZmán ${ }^{1}$ \\ 'Departamento de Biología Comparada, Facultad de Ciencias, Universidad Nacional Autónoma de México. \\ Apartado Postal 70-356, C.P. 04510, México, D.F., México. \\ ${ }^{2}$ Instituto de Ecología, A.C., Centro Regional del Bajío. Apartado Postal 386, C.P. 61600 Pátzcuaro, \\ Michoacán, México. \\ ${ }^{3}$ Autor para la correspondencia. Correo-e: sem@hp.fciencias.unam.mx
}

\begin{abstract}
Resumen: Con el propósito de contribuir al conocimiento embriológico del género Pinguicula (Lentibulariaceae) se estudiaron los procesos ontogenéticos de las estructuras reproductoras masculinas de una especie representativa de cada uno de los tres subgéneros que conforman a este género. Los caracteres embriológicos relacionados con el desarrollo de la pared de la antera, la microsporogénesis, la microgametogénesis y la morfología de los granos de polen se describen y fueron generalmente homogéneos entre las tres especies, lo cual refuerza la hipótesis de que es un grupo monofilético y no apoya la división del género Pinguicula en los subgéneros Isoloba, Temnoceras y Pinguicula propuestos por Casper (1966).
\end{abstract}

Palabras clave: granos de polen, Lentibulariaceae, microgametogenesis, microsporogenesis, Pinguicula.

Abstract: In order to gain greater knowledge about the embryology of the genus Pinguicula (Lentibulariaceae), a study of the development of male structures was conducted for a representative species of each subgenus. Embryological features concerning the development of the anther wall, microsporogenesis, microgametogenesis and pollen grains are described for the three species. These were generally consistent between them, and this evidence strongly supports the hypothesis that it is a monophyletic group. It does not support the division of the genus Pinguicula into three subgenera: Isoloba, Pinguicula and Temnoceras proposed by Casper (1966).

Key words: Lentibulariaceae, microsporogenesis, microgametogenesis, Pinguicula, pollen grain.

$\mathbf{L}$ a familia Lentibulariaceae está conformada por los géneros Genlisea, Pinguicula y Utricularia (FrommTrinta, 1979; Taylor, 1989, 1991; Zamudio, 1990; Olvera, 1996). Las más de 300 especies que la componen corresponden a plantas herbáceas, anuales o perennes, que pueden ser terrestres, acuáticas o epífitas (Gibson, 1974; Taylor, 1989; Zamudio, 1990). Son cosmopolitas y se distribuyen en las regiones templadas, tropicales (Juniper et al., 1989) y árticas (Taylor, 1989; Karlsson et al., 1991).

En México se encuentran representados los tres géneros: Pinguicula con 44 especies (Zamudio y Ludlow-Weichers, 1993; Zamudio, 2001), Utricularia con 19 especies
(Olvera, 1996, 1997), y Genlisea con una especie (Olvera y Martínez, 2002).

Con base en la morfología de la corola, el género Pinguicula se ha dividido en los subgéneros Isoloba, Pinguicula y Temnoceras (Casper, 1966). Todos ellos están representados en México, territorio en donde crece casi $50 \%$ de las especies de Pinguicula registradas a nivel mundial y donde más de $90 \%$ son consideradas endémicas (Zamudio, 1990).

La información embriológica existente de la familia Lentibulariaceae corresponde a investigaciones realizadas en el género Utricularia (Kausik, 1938; Khan, 1954; Kausik y Raju, 1955; Farooq y Siddiqui, 1964, 1967; 
Shivaramiah, 1964a,b, 1967; Begum, 1965; Farooq, 1965a,b, 1966; Farooq y Bilquis, 1966; Siddiqui, 1968, 1978a,b). Los géneros Pinguicula y Genlisea han sido poco estudiados; para el primero de ellos, la información de las estructuras femeninas es más abundante que de las masculinas (Stolt, 1936; Crété, 1956a, b; Haccius y HartlBaude, 1956; Kopczynska, 1964; Davis, 1966; FrommTrinta, 1979; Ibannain y Vallade, 1989; Nagendran y Dinesh, 1989; Johri et al., 1992). Aunado a esto, las investigaciones palinológicas también se han realizado principalmente en especies de Utricularia (Erdtman, 1952; Khan, 1954; Faegri e Iversen, 1964; Shivaramiah, 1964a, 1967; Begum, 1965; Thanikaimoni, 1966; Huynh, 1968; Casper y Manitz, 1975; Sohma, 1975a, b; Siddiqui, 1978a; Lobreau-Callen et al., 1999), mientras que en Pinguicula y Genlisea son escasas (Erdtman, 1952; Faegri e Iversen, 1964; Casper, 1966; Heusser, 1971; Sohma, 1975b; Fromm-Trinta, 1979, 1981; Zamudio y Lux, 1992; Zamudio y Ludlow-Weichers, 1993; Zamudio, 2001).

El propósito de la presente investigación fue estudiar el desarrollo de la pared de la antera, la microsporogénesis, la microgametogénesis y la morfología de los granos de polen de una especie mexicana representativa de los subgéneros Isoloba, Pinguicula y Temnoceras con el fin de contribuir al conocimiento embriológico del género Pinguicula y de la familia Lentibulariaceae.

\section{Materiales y métodos}

Material biológico. Se seleccionó a una especie mexicana representativa de cada subgénero. En cada caso los ejemplares de respaldo se depositaron en el herbario del Instituto de Ecología A.C., Centro Regional del Bajío (IEB).

(1) Pinguicula agnata Casper (subgénero Isoloba) es una especie perenne y heterófila. La floración y la fructificación se presentan de septiembre a noviembre. Las plantas crecen sobre rocas sedimentarias, calcáreas o lutitas. Las poblaciones colectadas se ubican en el arroyo Tolimán, municipio de Zimapán, Hidalgo (2049.344' N; 99²6.225' O) a 1,150 m snm, y en la cañada de La Culebra, municipio de Cadereyta, Querétaro (2056’18' N; 9940’38'” O) a 1,800 m snm. La vegetación de estos sitios corresponde a un matorral desértico con elementos de bosque tropical caducifolio (Rzedowski, 1978).

Especímenes: Sergio Zamudio y E. Pérez-Calix 9517 y 9771.

(2) Pinguicula oblongiloba DC. (subgénero Pinguicula) es una especie perenne y heterófila. La floración y la fructificación se presentan de mayo a agosto. Las plantas crecen en suelos arcillosos rojizos o café-rojizos, derivados de rocas ígneas extrusivas. Los ejemplares se colectaron en la ladera NO del Cerro Blanco, municipio de Pátzcuaro, Michoacán (19³1'57' N, 101³6'03”'O) entre 2,150 y $2,200 \mathrm{~m} \mathrm{snm}$. Esta es un área perturbada y erosionada con remanentes de la vegetación original formada por un bosque de pino-encino (Rzedowski, 1978).

Especímenes: Sergio Zamudio 9520, 9522, 9517 y 9817.

(3) Pinguicula crenatiloba DC. (subgénero Temnoceras) es una especie anual y homófila. Las flores y frutos aparecen de agosto a diciembre. Las plantas crecen en suelos arcillosos rojizos o café-rojizos, derivados de rocas ígneas extrusivas. Se colectaron en la loma de Santa María, al sur de Morelia, Michoacán (1941' N, $101^{\circ} 11.3^{\prime}$ O) a 2,300 m snm, y en la ladera NO del Cerro Blanco, municipio de Pátzcuaro, Michoacán (19³1'57', N, 101 36 '03'” O) entre 2,150 y 2,200 m snm, sobre musgo y en sitos abiertos del bosque de pino-encino (Rzedowski, 1978).

Especímenes: Sergio Zamudio 9,564, 10,151. Sergio Zamudio y E. Pérez 9,246.

Técnica histológica. De cada especie se recolectaron botones florales en diferentes etapas de desarrollo y flores en antesis. El material recolectado se fijó en el sitio de la colecta utilizando FAA (formol, ácido acético, etanol 96\%, agua 2:1:10:7). Se lavó con agua corriente y se deshidrató en una serie gradual de etanol. Posteriormente el material se incluyó en la resina JB-4 (Ruzin, 1999) y se cortó en un ultramicrotomo Sorvall MT2-B a $2 \mu \mathrm{m}$ de grosor. Los cortes se tiñeron con azul de toluidina acuosa al 1\%. Las laminillas se observaron y fotografiaron en un microscopio óptico Olympus Provis AX-70.

Técnica de acetolisis ácida. Sólo los granos de polen de Pinguicula agnata fueron acetolizados y montados en jalea glicerinada de acuerdo con la técnica de Erdtman (1952). Se midió el grosor de la sexina y nexina, así como el diámetro polar y ecuatorial, para obtener la relación del primero sobre el segundo (P/E) de 20 granos de polen de cinco ejemplares procesados, y se obtuvieron los promedios.

Microscopía electrónica de barrido (MEB). Granos de polen maduros y flores en antesis de cada una de las especies fueron colocados en portamuestras de aluminio sobre cinta conductiva de carbón de doble cara. Se cubrieron con una capa de oro en una ionizadora Denton Vacuum Desk II. Se observaron y fotografiaron en un microscopio electrónico de barrido Jeol 35 y Jeol JSM-5310LV.

\section{Resultados}

Dado que el patrón de desarrollo del androceo es similar y la morfología de los granos presenta pocas diferencias en las tres especies estudiadas, sólo se describirá el desarrollo de Pinguicula agnata y se harán comentarios adicionales 

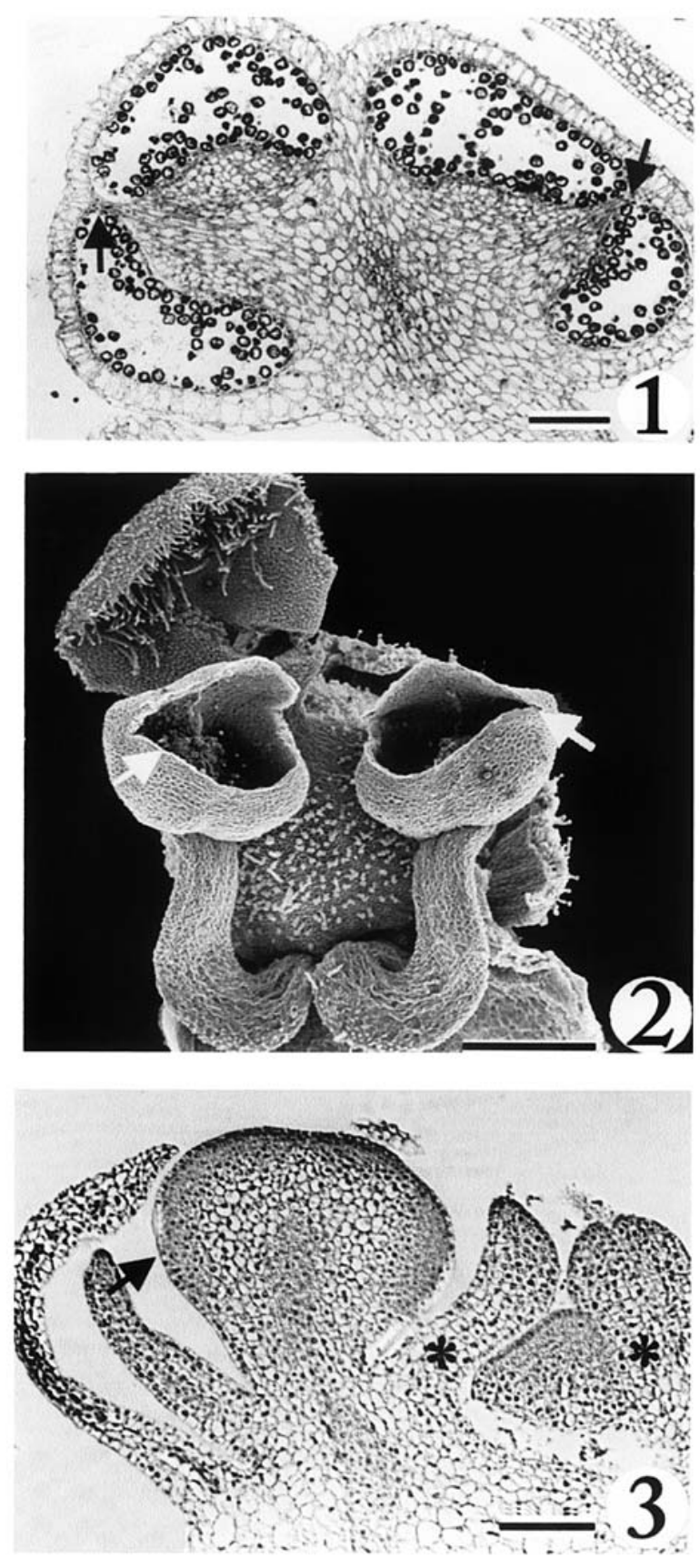

Figuras 1-3. Anatomía y morfología del androceo. 1. Corte transversal de antera mostrando los microsporangios o sacos polínicos separados por el septo (flechas); barra $=54 \mu \mathrm{m}$. 2. Flor en antesis mostrando la antera madura unilocular (flechas); barra $=500 \mu \mathrm{m}$. 3. Corte longitudinal de botón floral mostrando un primordio de la antera (flecha) y los carpelos (asteriscos); barra $=100 \mu \mathrm{m}$. para $P$. oblongiloba y $P$. crenatiloba cuando se presente alguna diferencia.

En preantesis las anteras son bilobadas y tetrasporangiadas (figura 1), pero en la antesis la antera es unilocular debido a que el tejido conectivo y el septo se degradan. La dehiscencia de las anteras es extrorsa (figura 2).

Desarrollo de la pared de la antera. Los verticilos florales emergen casi al mismo tiempo, pero son los primordios de las anteras los que crecen y se diferencian más rápido (figura 3). Una vez que la protodermis se ha diferenciado en la epidermis, el tejido arquesporial se divide periclinalmente, originando a la capa parietal primaria y al tejido esporógeno (figura 4). Como resultado de la división periclinal de la capa parietal primaria, se originan las capas parietales secundarias interna y externa (figura 5). La primera se diferencia en las células del tapete y la segunda se divide nuevamente para dar origen a las células del endotecio y la capa media (figura 6). De esta forma, el desarrollo de la pared de la antera corresponde al tipo dicotiledóneo (sensu Davis, 1966), y consiste de cuatro capas uniestratificadas: la epidermis, el endotecio, la capa media y el tapete secretor uni-binucleado. Las células del tejido conectivo, adyacentes al esporangio, adquieren la apariencia de células tapetales, son uni-binucleadas con citoplasma denso (figuras 7 y 10).

Microsporogénesis y microgametogénesis. El tejido esporógeno se diferencia en las células madres de las microsporas, las cuales se rodean de calosa (figura 7). Al término de la meiosis, se originan tétradas tetraédricas de microsporas rodeadas también por calosa (figura 8); una vez que ésta se degrada, las microsporas uninucleadas o granos de polen jóvenes son liberados (figura 9). En esta etapa, las células tapetales reducen su contenido citoplásmico y el grosor de las paredes celulares, mientras que las células de la capa media se colapsan y adosan al endotecio (figura 10). Cuando finaliza la actividad celular de las células tapetales, los restos del citoplasma son liberados al interior de los esporangios. En esta etapa, algunas células del endotecio de Pinguicula crenatiloba se dividen periclinalmente, dando lugar a una capa parcialmente biestratificada. Este fenómeno no se observó en $P$. oblongiloba ni en $P$. agnata (figura 11).

Los granos de polen se liberan como mónadas en etapa bicelular (figura 12); en esta etapa la pared de la antera consiste de la epidermis y del endotecio cuyas células se han elongado radialmente y muestran engrosamientos en forma de barra en las paredes anticlinales y tangenciales internas (figura 13).

Morfología de los granos de polen. En esta investigación sólo los granos de polen de P. agnata se estudiaron con microscopia óptica y MEB. $P$. oblongiloba y P. crenatiloba 

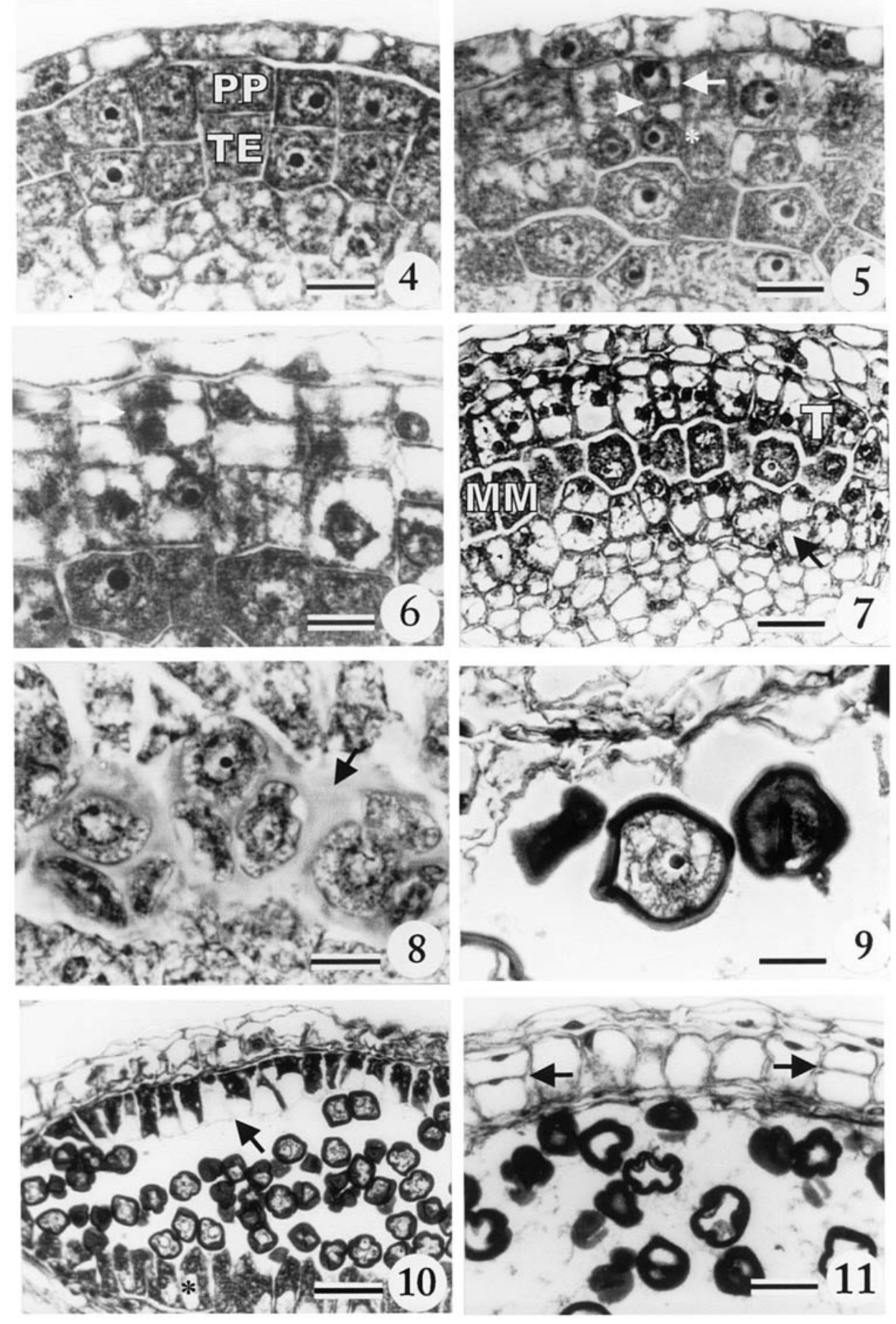

Figuras 4-11. Desarrollo de la pared de antera, microsporogénesis y microgametogénesis en Pinguicula agnata. 4. Se muestra a la capa parietal primaria (PP) y al tejido esporógeno (TE) resultado de la división periclinal de las células arquesporiales; barra $=10 \mu \mathrm{m}$. 5. Se observa la división de la capa parietal primaria (cabeza de flecha) para dar origen a la capa parietal secundaria interna (flecha) y externa (asterisco); barra $=10 \mu \mathrm{m}$. 6. Se muestra la división periclinal de la capa parietal secundaria externa (flecha); barra $=11 \mu \mathrm{m}$. 7. Pared de la antera mostrando las capas que la conforman. Se observa al tapete (T) rodeando a las células madres de las microsporas (MM). La flecha indica al tapete que se ha originado del tejido conectivo; barra $=53 \mu \mathrm{m}$. 8. Tétrada tetraédrica de microspora rodeada por la pared de calosa (flecha); barra $=55 \mu \mathrm{m}$. 9. Microspora uninucleada; barra $=8 \mu \mathrm{m}$. 10. Antera al final de la meiosis mostrando el tapete secretor (flecha), el asterisco indica el tapete de origen conectivo; barra $=27 \mu \mathrm{m}$. 11. $P$. crenatiloba se muesta la capa del endotecio parcialmente biestratificada (flechas); barra $=51 \mu \mathrm{m}$. 

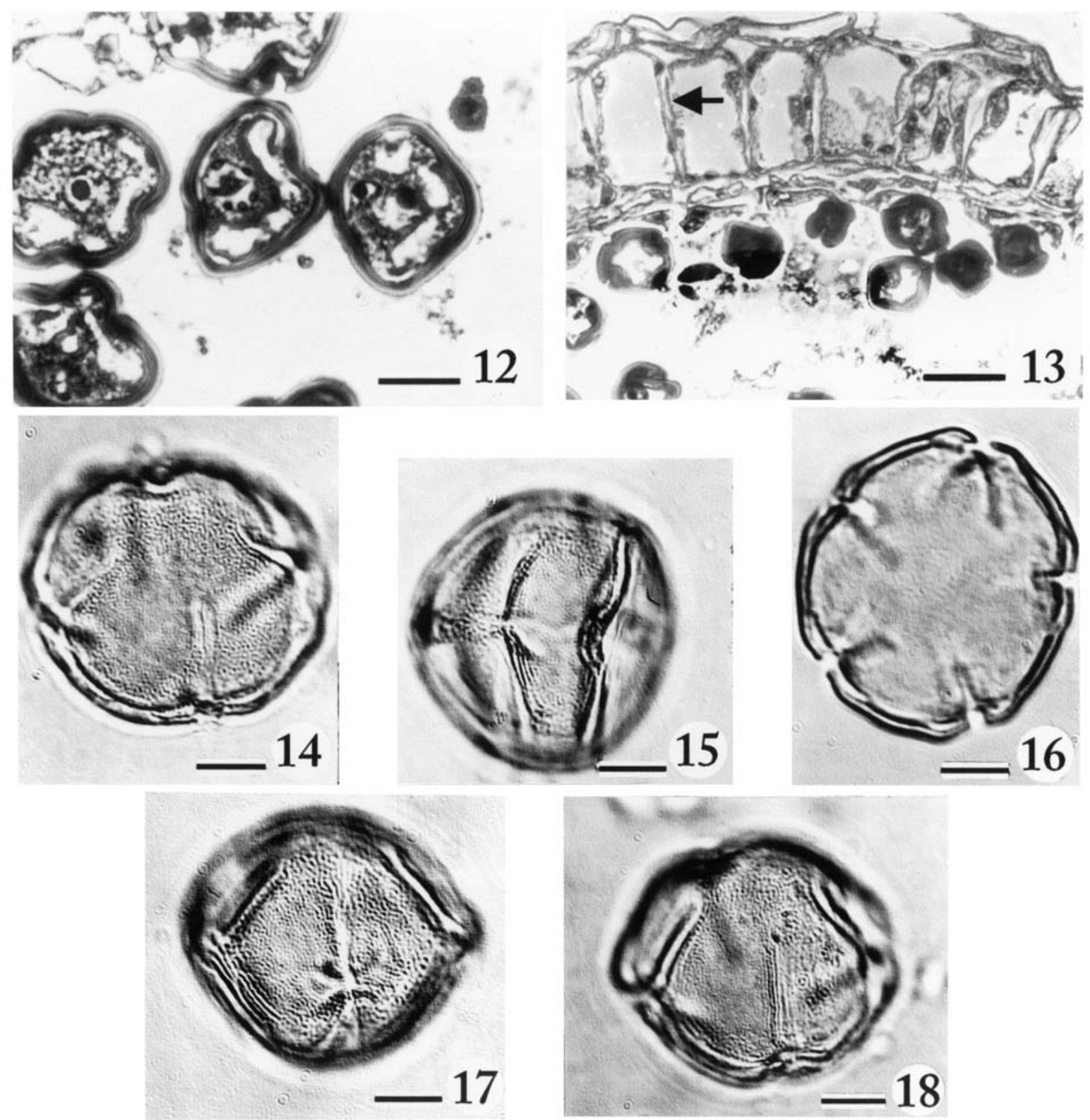

Figuras 12-18. Pinguicula agnata. Microgametogénesis y pared de antera (figs. 12-13). Morfología de los granos de polen (figs. 14-18). 12. Grano de polen en etapa bicelular mostrando la célula vegetativa y la célula generatriz; barra $=10 \mu \mathrm{m}$. 13. Pared de antera madura, se muestra los engrosamientos del endotecio (flecha); barra $=51 \mu \mathrm{m}$. 14. Grano de polen tetracolporado mostrando la vista polar circular con cuatro lóbulos; barra $=9 \mu \mathrm{m}$. 15. Grano de polen prolado en vista ecuatorial; barra $=9 \mu \mathrm{m}$. 16. Grano de polen pentacolporado mostrando la vista polar circular con cinco lóbulos; barra $=9 \mu \mathrm{m}$. 17. Grano de polen en vista ecuatorial mostrando una endoabertura; barra $=9 \mu \mathrm{m}$. 18. Grano de polen en vista ecuatorial mostrando la exina tectada reticulada; barra $=9 \mu \mathrm{m}$. 

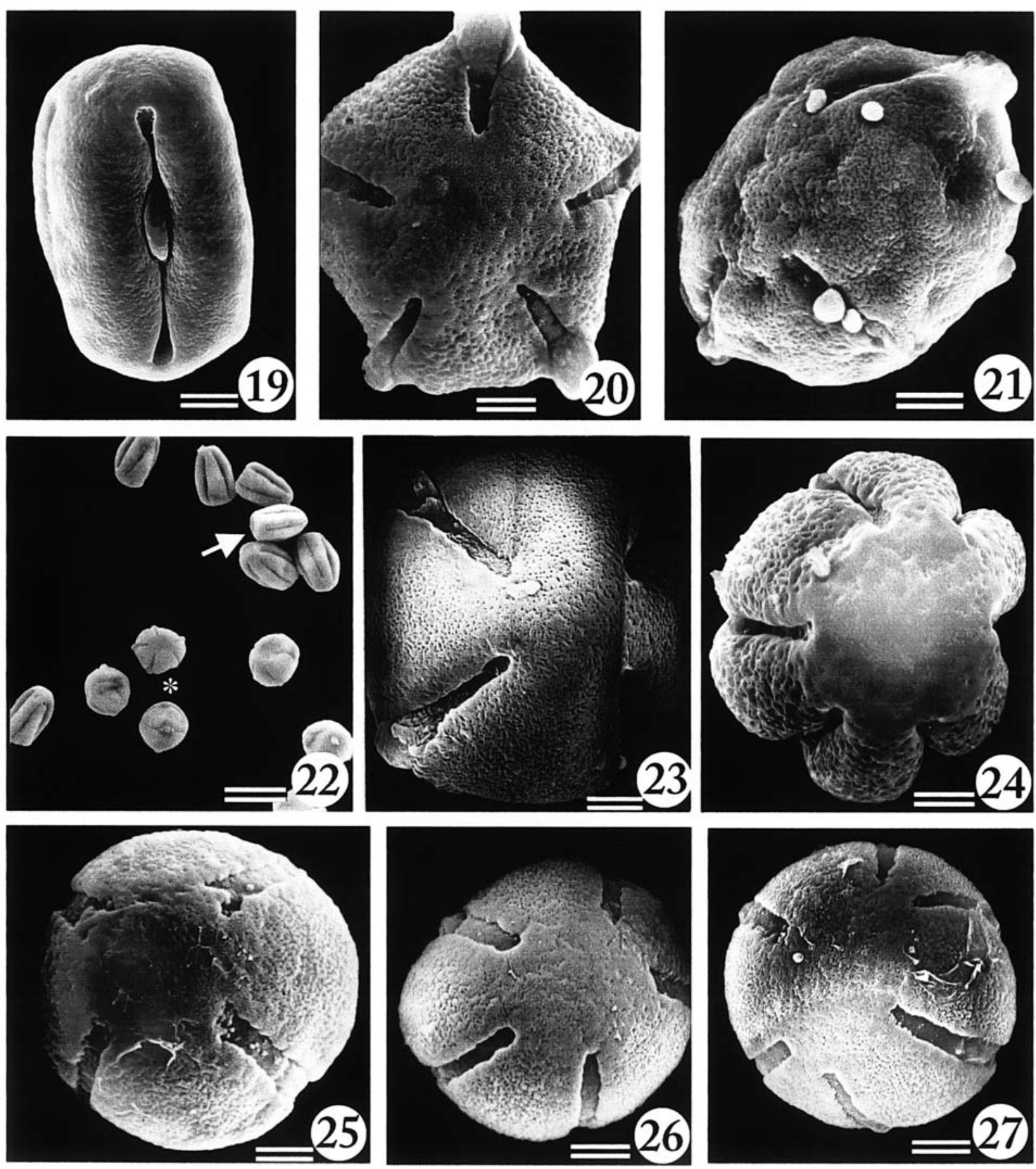

Figuras. 19-27. Granos de polen con microscopía electrónica de barrido. Pinguicula agnata (figs. 19-21), P. oblongiloba (figs. 22-24) y P. crenatiloba (figs. 25-27). 19. Grano de polen prolado tetracolporado en vista ecuatorial; barra $=4 \mu \mathrm{m}$. 20. Grano de polen pentacolporado mostrando la vista polar pentagonal, nótese la exina microreticulada; barra $=4 \mu \mathrm{m}$. 21. Grano de polen de forma anormal; barra $=4 \mu \mathrm{m}$. 22. Vista panorámica mostrando granos de polen prolados (flecha) y oblados (asterisco); barra $=3 \mu \mathrm{m}$. 23. Grano de polen tetracolporado mostrando la vista polar cuadrangular, se observa la exina microreticulada; barra $=5 \mu \mathrm{m}$. 24. Grano de polen hexacolporado mostrando la vista polar circular con seis lóbulos y exina tectada microreticulada en la región polar y tectada reticulada en la región ecuatorial; barra $=6 \mu \mathrm{m}$. 25. Grano de polen tetracolporado; en vista polar circular con cuatro lóbulos; barra $=3 \mu \mathrm{m}$. 26. Grano de polen pentacolporado en vista polar circular con cinco lóbulos; barra $=5 \mu \mathrm{m}$. 27. Grano de polen hexacolporado mostrando la vista polar con seis lóbulos; barra $=4 \mu \mathrm{m}$. 
se estudiaron únicamente con MEB debido a que la descripción palinológica con microscopia óptica fue realizada por Zamudio y Ludlow-Weichers (1993).

Con microscopia óptica los granos de polen de $P$. agnata mostraron ser oblados (figura 14) y prolados (figura 15) de P: $35.25 \times$ E: $35.37 \mu \mathrm{m}$ y P: $25.16 \times$ E: $34.24 \mu \mathrm{m}$, respectivamente. Los granos de polen tienen vista polar con cuatro o cinco lóbulos (figura 16), y son tetra a pentacolporados, con largas ectoaberturas y membrana lisa (figura 17). El grosor de la sexina y nexina es de $1 \mu \mathrm{m}$. La exina es tectada, reticulada, de $2 \mu \mathrm{m}$ de grosor (figura 18).

Con el MEB se confirmó la presencia de granos de polen oblados y prolados, mientras que la exina se observó tectada reticulada en la región ecuatorial y tectada microreticulada en la región polar. La región ecuatorial puede ser rectangular (figura 19), mientras que la región polar puede ser cuadrada o pentagonal (figura 20), con cuatro o cinco y rara vez seis lóbulos. Aproximadamente $20 \%$ de los granos de polen de $P$. agnata mostraron anormalidades morfológicas y grupos de éstos permanecen aún rodeados por restos de la pared de calosa (figura 21).

En P. oblongiloba, con MEB también se observaron granos de polen oblados, prolados y aproximadamente $40 \%$ con anormalidades en sus formas, así como rodeados por restos de la pared de calosa (figura 22). La región polar de los granos de polen oblados es circular con cinco lóbulos, mientras que la región polar de los prolados es cuadrada con cuatro lóbulos (figura 23). Se observaron granos de polen tetra a pentacolporados con largas ectoaberturas, mientras que los granos de polen hexacolporados fueron escasos. La exina es tectada reticulada en la región ecuatorial y tectada microreticulada en la región polar (figura 24).

En P. crenatiloba $80 \%$ de los granos de polen son oblados y $15 \%$ son prolados. En ambos tipos la vista polar es circular con cuatro a seis lóbulos (figuras 25-27). Son tetra a hexacolporados con exina tectada reticulada en la región ecuatorial y microreticulada en la región polar. Sólo 5\% de los granos de polen observados mostraron anormalidades en su forma.

\section{Discusión}

En el género Pinguicula sólo se presentan dos estambres anteriores (Casper, 1966), y aunque excepcionalmente se ha registrado la existencia de un número mayor, éstos pueden ser el resultado de anormalidades en el desarrollo de este verticilo (Wydler, 1857; Dickson, 1869). En ninguna de las más de 100 flores examinadas de Pinguicula agnata, $P$. oblongiloba y $P$. crenatiloba se observaron más de dos estambres funcionales o estaminodios. La presencia de dos estambres anteriores es un carácter constante en la familia, el cual ha sido registrado también en Genlisea y Utricularia (Fromm-Trinta, 1979; Taylor, 1989).

Con respecto al orden Lamiales, Pinguicula se caracteri- za por poseer ontogenéticamente sólo dos estambres con anteras secundariamente uniloculares, por la degradación del tejido conectivo y del septo, como lo mostraron Casper (1966) y las observaciones de esta investigación. Esto explicaría el hecho de que Barnhart (1916) haya observado anteras tanto monoesporangiadas como biesporangiadas en $P$. vulgaris. En los otros géneros no se ha observado la degradación de ningún tejido (Davis, 1966; Johri et al., 1992). En Genlisea las anteras son exclusivamente biesporangiadas (Fromm-Trinta, 1979), mientras que Utricularia presenta anteras tetraesporangiadas (Khan, 1954; Farooq, 1966; Shivaramiah, 1967; Siddiqui, 1978b), pentaesporangiadas (Khan, 1954) y hexasporangiadas (Siddiqui, 1978b).

Desarrollo de la pared de la antera. En general, el tipo de desarrollo de la pared de la antera es un estado de carácter que se desconoce para la mayoría de las especies de Lamiales. En éstas sólo se señalan las características de las capas de la pared de la antera, una vez que las divisiones del tejido parietal han concluido. En las especies aquí estudiadas el desarrollo de la pared de la antera es exclusivamente del tipo dicotiledóneo, lo cual contrasta con Utricularia, que se caracteriza por presentar tres tipos de desarrollo de la pared de antera: el dicotiledóneo (Kausik, 1938; Khan, 1954; Kausik y Raju, 1955; Farooq y Siddiqui, 1964, 1967; Farooq, 1966; Siddiqui, 1978a), el monocotiledóneo (Begum, 1965; Farooq, 1965a) y el básico (Siddiqui, 1978a,b), con la presencia simultánea del tipo básico y dicotiledóneo en $U$. dichotoma (Siddiqui, 1978a).

Generalmente las capas de la pared de la antera en Lamiales son uniestratificadas, aunque se pueden presentar dos o más estratos, como sucede en el endotecio y las células tapetales; estas últimas además presentan un doble origen en Scrophulariacerae, Bignoniaceae, Oleace, Orobanchaceae y Lentibulariaceae (Farooq, 1965a,b 1966; Farooq y Siddiqui, 1967; Siddiqui, 1978a,b; Johri et al., 1992). En Lentibulariaceae, el doble origen del tejido tapetal se ha observado en Pinguicula y Utricularia; en Genlisea esta información se desconoce. Con respecto al endotecio, sólo P. crenatiloba presentó un endotecio parcialmente biestratificado, estructura no descrita con anterioridad para el género y que comparte con $U$. dichotoma (Siddiqui, 1978a) y U. cornuta (Siddiqui, 1978b). En el caso de la capa media, ésta presenta de dos a cuatro estratos en Scrophulariaceae, Bignoniaceae y Orobanchaceae (Johri et al., 1992), lo cual no se ha registrado en ningún miembro de la familia Lentibulariaceae.

El tapete de tipo secretor se presenta en todas las familias de Lamiales y es una característica embriológica del orden. Sin embargo, en Utricularia scandens ocasionalmente las paredes de las células tapetales se rompen y el citoplasma de estas células forma un coenocito dentro del lóculo (Farooq y Bilquis, 1966). Es necesario confirmar esta observación, ya que esto representaría el primer registro de tapete 
de tipo plasmodial en Lentibulariaceae y sería la única familia en el orden en presentar este estado de carácter.

Microsporogénesis y microgametogénesis. De acuerdo con Johri et al. (1992), en las familias del orden Lamiales la microsporogénesis es exclusivamente del tipo simultáneo. La única excepción a este hecho se presenta en Lentibulariaceae con Pinguicula, que presenta el tipo sucesivo, y Utricularia, que se caracteriza por presentar los dos tipos conocidos de microsporogénesis, el sucesivo y el simultáneo (Khan, 1954; Farooq, 1966; Shivaramiah, 1967; Siddiqui, 1978a,b). Aunado a esto, la microsporogénesis en este género puede ser un proceso asincrónico, pudiendo presentarse en un mismo esporangio células madres de las microsporas, tétradas de microsporas y microsporas libres (Farooq, 1965b, 1966; Farooq y Bilquis, 1966; Siddiqui, 1978a,b).

Al término de la microsporogénesis Pinguicula presenta únicamente tétradas tetraédricas de microsporas, lo cual contrasta fuertemente con Utricularia (Khan,1954; Farooq y Siddiqui, 1964; Shivaramiah, 1964a,b, 1967; Begum, 1965; Farooq, 1965a,b, 1966; Farooq y Bilquis, 1966; Farooq y Siddiqui, 1967; Siddiqui, 1968, 1978a,b) y el resto del orden Lamiales (Johri et al., 1992), en donde también se han observado tétradas isobilaterales, en forma de $\mathrm{T}$, decusadas y romboidales. Sólo para algunas especies de Utricularia se ha mencionado que en un mismo esporangio coexisten más de un tipo de tétrada de microspora (Farooq y Bilquis, 1966; Farooq y Siddiqui, 1967).

En el orden Lamiales lo común es la dispersión de los granos de polen en etapa bicelular (Johri et al., 1992), lo cual contrasta con el género Utricularia, cuyos granos de polen se dispersan en etapa tricelular (Kausik, 1938; Khan, 1954; Kausik y Raju, 1955; Farooq y Siddiqui, 1964; Shivaramiah, 1964a,b, 1967; Farooq, 1965a,b, 1966; Farooq y Bilquis, 1966; Farooq y Siddiqui, 1967; Siddiqui, 1968, 1978a,b).

Morfología de los granos de polen. Las formas de los granos de polen observadas en esta investigación concuerdan con lo descrito para la familia Lentibulariaceae (Erdtman, 1952; Faegri e Iversen, 1964; Sohma, 1975a,b; FrommTrinta, 1979; Zamudio y Ludlow-Weichers, 1993; Berger, 1998 [trabajo inédito]; Sohma, 1975a,b). Por otro lado, la morfología de los granos de polen es similar en las especies de Pinguicula aquí estudiadas. Éstos son oblados y prolados, tetra a hexacolporados, con exina tectada microrreticulada a tectada reticulada. Esta descripción coincide con las observaciones en otras especies de este mismo género (Erdtman, 1952; Casper, 1966; Heusser, 1971; Sohma, 1975b; Zamudio y Lux, 1992; Zamudio y LudlowWeichers, 1993).

En $P$. vulgaris y $P$. ramosa también se presentan granos de polen con diferencias en la ornamentación de la exina
(Sohma, 1975b), como fue observado en las tres especies de esta investigación, caracter que no se ha observado en Genlisea (Erdtman, 1952; Fromm-Trinta, 1979, 1981) ni en Utricularia (Thanikaimoni, 1966; Sohma, 1975a; Taylor, 1989).

En general, la exina en las especies de Pinguicula es tectada reticulada a finamente reticulada (Casper, 1966; Heusser, 1971; Sohma, 1975b; Zamudio y Lux, 1992; Zamudio y Ludlow-Weichers, 1993), ocasionalmente verrugada, psilada escabrada (Sohma, 1975b) o puntitegilada (Zamudio, 2001). Por otro lado, en Genlisea sólo se ha registrado exina subreticulada (Erdtman, 1952) y tectada rugosa (Fromm-Trinta, 1979, 1981). A diferencia de los géneros antes mencionados, Utricularia muestra la mayor diversidad de ornamentaciones de la exina. Las primeras investigaciones mostraron que ésta era tectada lisa, por lo que fueron considerados como homogéneos (Kausik, 1938; Shivaramiah, 1964a,b, 1967; Begum, 1965; Farooq, 1965a; Siddiqui, 1978a). Posteriormente se han observado otros tipos como tectada finamente reticulada (Erdtman, 1952; Khan, 1970; Sohma, 1975a; Johri et al., 1992), tectada escabrada (Thanikaimoni, 1966), tectada psilada a escabrada (Thanikaimoni, 1966; Sohma, 1975a), puntitectada (Casper y Manitz, 1975) y microequinada con espinas agudas (Lobreau-Callen et al., 1999).

A nivel del orden Lamiales, el carácter básico es la presencia de granos de polen tricolpados o tricolporados. Entre los miembros de la familia Lentibulariaceae, la diferencia más importante radica en el número de las aberturas en los granos de polen. En Genlisea y Pinguicula éstos generalmente son tricolporados a rara vez octacolporados (Erdtman, 1952; Casper, 1966; Heusser, 1971; FrommTrinta, 1979, 1981; Sohma, 1975b; Zamudio y Lux, 1992; Zamudio y Ludlow-Weichers, 1993; Berger, 1998 [trabajo inédito]; Zamudio, 2001). En contraste, en Utricularia son raras las especies que presentan granos de polen tri-tetracolporados, ya que la mayoría presentan de 5 a 28 aberturas, y generalmente son sincolpados a sinorados (Thanikaimoni, 1966; Huynh, 1968; Casper y Manitz, 1975; Sohma, 1975a,b; Taylor, 1989; Berger, 1998 [trabajo inédito]; Lobreau-Callen et al., 1999). Por lo tanto, la morfología de los granos de polen en Utricularia es extremadamente diversa y sería deseable realizar estudios más detallados para Pinguicula y Genlisea, géneros para los que en esta información aún está incompleta.

En Pinguicula (Casper, 1966; Heusser, 1971; Sohma, 1975b; Zamudio y Lux, 1992; Zamudio y LudlowWeichers, 1993; Zamudio, 2001) no se ha establecido ninguna relación entre el hábitat, la morfología vegetativa y la morfología polínica como se ha hecho en Utricularia (Thanikaimoni, 1966; Huynh, 1968; Taylor, 1989). De acuerdo con Lobreau-Callen et al. (1999), la variación en el polen está relacionada con la historia evolutiva de cada especie y no con la especialización del hábitat, y por lo 
tanto no es factible establecer este tipo de relaciones.

En la familia Lentibulariaceae, sólo en Utricularia se presenta la cleistogamia y la germinación de los granos de polen en el interior de las anteras (Farooq, 1965a,b, 1966; Farooq y Bilquis, 1966; Farooq y Siddiqui, 1967; Taylor, 1989), procesos que podrían estar asociados con el hábito acuático. Estos aspectos son desconocidos para los géneros Pinguicula y Genlisea y requieren de estudios detallados.

Los caracteres embriológicos aquí observados sugieren que en la familia Lentibulariaceae, Pinguicula es el género que conserva un mayor número de caracteres embriológicos ancestrales como son la microsporogénesis sucesiva, la presencia de tétradas tetraédricas, los granos de polen maduros que se dispersan en etapa bicelular y la presencia de un menor número de aberturas en los granos de polen.

\section{Conclusiones}

A pesar de las diferencias morfológicas que se observan en la flor de cada uno de los subgéneros del género Pinguicula, los caracteres embriológicos masculinos son homogéneos. Esto refuerza la hipótesis de que éste es un grupo monofilético, como lo proponen Albert et al. (1992) y Jobson et al. (2003) con el uso de marcadores moleculares. En cambio, los caracteres embriológicos no apoyan la división del género Pinguicula en los subgéneros propuestos por Casper (1966).

\section{Agradecimientos}

Agradecemos a Beatriz Ludlow-Wiechers por la asesoría en la descripción de los granos de polen; a Yolanda Hornelas del Instituto de Ciencias del Mar y Limnología (UNAM) por las fotografías en el microscopio electrónico de barrido; a Alejandro Martínez Mena y Alfredo Gamboa Romero del Laboratorio de Microcine de la Facultad de Ciencias (UNAM), por la toma de las imágenes fotográficas y por el procesamiento de los negativos respectivamente; y a Carlos Guido Formosa de la ENP Plantel 8 (UNAM), por la digitalización y manejo de las imágenes. Esta investigación fue apoyada por la Dirección General de Asuntos del Personal Académico y el Programa de Apoyo a las Divisiones de Estudios de Posgrado de la Universidad Nacional Autónoma de México.

\section{Literatura citada}

Albert V.A., Williams S.E. y Chase M.W. 1992. Carnivorous plants: phylogeny and structural evolution. Science 257:14911495.

Barnhart J.H. 1916. Segregation of genera in Lentibulariaceae. Memoirs of the New York Botanical Garden 6:39-64.

Begum M. 1965. Studies on the embryology of Utricularia graminifolia Vahl. Current Science 34:355-356.

Berger D. 1998. Pollenmorphologische Untersuchungen
Cubanischer Lentibulariaceae. Institut für Spezielle Botanik, Humboldt Universität zu Berlin, Alemania. 21 pp. [Trabajo inédito].

Casper J.S. 1966. Monographie der Gattung Pinguicula L. Bibliotheca Botanica 31:1-209.

Casper J.S y Manitz H. 1975. Beiträge zur Taxonomie and Chorologie der mitteleuropäischen Utricularia-Arten. Feddes Repertorium 86:211-232.

Crété P. 1956a. Lentibulariacées. Développement de l'embryon chez le Pinguicula leptoceras Rchb. Comptes Rendus Hebdomadairesdes Séances de l'Académie des Sciences Paris 242:1063-1066.

Crété P. 1956b. Lentibulariacées. Développement de l'albumen chez les Pinguicula. Comptes Rendus Hebdomadairesdes Séances de l'Académie des Sciences Paris 242:1346-1349.

Davis G.L. 1966. Systematic embryology of the angiosperms. John Wiley and Sons, Nueva York.

Dickson A. 1869. On the development of the flower of Pinguicula vulgaris L., with remarks on the embryos of $P$. vulgaris, $P$. grandiflora, P. lusitanica, $P$. caudata and Utricularia minor. Transactions of the Royal Society of Edinburgh 25:639-653.

Erdtman G. 1952. Pollen Morphology and Plant Taxonomy. Angiosperms. Almqvist and Wiksel, Estocolmo.

Faegri K. e Iversen J. 1964. Textbook of Pollen Analysis. Blackwell, Oxford.

Farooq M. 1965a. Studies in the Lentibulariaceae II. The embryology of Utricularia arcuata Wt. Journal of the Indian Botanical Society 44:326-346.

Farooq M. 1965b. Studies in the Lentibulariaceae III. The embryology of Utricularia uliginosa Vahl. Phytomorphology 15:123131.

Farooq M. 1966. Studies in the Lentibulariaceae IV. The embryology of Utricularia striatula Sm. Journal of the Indian Botanical Society 45:1-13.

Farooq M. y Bilquis S. 1966. Studies in the Lentibulariaceae VIII. The life history of Utricularia scandens Benj. Beiträge zur Biologie der Pflanzen. 42:363-371.

Farooq M.S. y Siddiqui S.A. 1964. The embryology of Utricularia stellaris Linn.f. Science and Culture 30:394-395.

Farooq M.S. y Siddiqui S.A. 1967. Studies in the Lentibulariaceae VI. The embryology of Utricularia stellaris Linn. Journal of the Indian Botanical Society 46:31-44.

Fromm-Trinta E. 1979. Revisão das espécies do gênero Genlisea St-Hill. (Lentibulariaceae) das regiões sudeste e sul do Brasil. Rodriguésia 31:17-139.

Fromm-Trinta E. 1981. Revisão do gênero Genlisea St-Hill. (Lentibulariaceae) do Brasil. Boletim do Museu Nacional de Rio de Janeiro n.s., Botanica 61:1-21.

Gibson D.N. 1974. Lentibulariaceae. Bladderwort Family. En: Standley P.C., Williams L.O y Gibson D.N. Eds. Flora de Guatemala, Fieldiana Botany 24, Part X(3-4):315-328.

Haccius B. y Hartl-Baude E. 1956. Embryologische und histogenetische Studien an "Monokotylen-Dikotylen". II. Pinguicula vulgaris L. und Pinguicula alpina L. Oesterreichische Botanische Zeitschrift 103:567-587.

Heusser C.J. 1971. Pollen and spores of Chile. The University of Arizona Press. Tucson.

Huynh K.L. 1968. Étude de la morphologie du pollen du genre Utricularia L. Pollen et Spores 10:11-55.

Ibannain Z. y Vallade J. 1989. Interprétation morphologique des 


\section{Silvia Espinosa-Matías, Sergio Zamudio y Judith Márquez-GuZmán}

embryons monocotylés. I. La localisation du territoire précaulinaire et la valeur morphologique du dicotylédon. Bulletin de la Société Botanique de France 136:305-316.

Jobson R.W., Playford J., Cameron K.M. y Albert V.A. 2003. Molecular phylogenetics of Lentibulariaceae inferred from Plastid rps16 Intron and trnL-F DNA sequences: implications for character evolution and biogeography. Systematic Botany 28:157-171.

Johri B.M., Ambegaokar K.B. y Srivastava P.S. 1992. Comparative Embryology of Angiosperms. Springer, Berlín.

Juniper B.E., Robins R.J y Joel D.M. 1989. The Carnivorous Plants. Academic Press, Londres.

Karlsson P.S., Nordell K.O., Carlsson B.A. y Svensson B.M. 1991. The effect of soil nutrient status on prey utilization in four carnivorous plants. Oecologia 86:1-7.

Kausik S.B. 1938. Pollen development and seed formation in Utricularia coerulea L. Beihefte Botanisches Centralblatt 58:365-378.

Kausik S.B. y Raju M.V.S. 1955. A contribution to the floral morphology and embryology of Utricularia reticulata Smith. Proceedings of the Indian Academy of Science 41:155-166.

Khan R. 1954. A contribution to the embryology of Utricularia flexuosa Vahl. Phytomorphology 4:80-117.

Khan R. 1970. Lentibulariaceae. Bulletin of the Indian National Academy of Science 41:290-297.

Kopczynska K. 1964. Embryosac-development in Pinguicula vulgaris L. Acta Societatis Botanicorum Poloniae 33:141-156.

Lobreau-Callen D., Jérémie J. y Suárez-Cervera M. 1999. Morphologie et ultrastructure du pollen dans le genre Utricularia L. (Lentibulariaceae). Canadian Journal of Botany 77:744-767.

Nagendran C.R. y Dinesh M.S. 1989. The Embryology of Angiosperms: A Classified Bibliography (1965-1985). Indira Publishing House, West Bloomfield, Michigan.

Olvera G.M. 1996. El Género Utricularia en México. Anales del Instituto de Biología Universidad Nacional Autónoma de México, Serie Botánica 67:347-384.

Olvera G.M. 1977. Primer registro de Utricularia erectiflora (Lentibulariaceae) para México. Anales del Instituto de Biología, Universidad Nacional Autónoma de México, Serie Botánica 62: 43-45.

Olvera G.M. y Martínez S.E. 2002. Primer registro de Genlisea (Lentibulariaceae) para México. Acta Botanica Mexicana 59:71-73.

Ruzin S.E. 1999. Plant Microthechnique and Microscopy. Oxford University Press, Oxford.

Rzedowski J. 1978. Vegetación de México. Limusa, México, D.F.
Shivaramiah G. 1964a. A contribution to the embryology of Utricularia exoleta R. Current Science 33:501-503.

Shivaramiah G. 1964b. A contribution to the embryology of Utricularia wallichiana Wt. Current Science 33:657-658.

Shivaramiah G. 1967. Observations on the floral morphology and embryology of Utricularia stricticaulis Stapf. Proceedings of the Indian Academy of Science 65:56-62.

Siddiqui S.A. 1968. Embryology of Utricularia reticulata var. parviflora Santapau and Utricularia nivea Valh Enum. Science and Culture 34:422-424.

Siddiqui S.A. 1978a. Studies in the Lentibulariaceae 8. The development of gametophytes in Utricularia dichotoma Labill. Flora (Jena) 167:111-116.

Siddiqui S.A. 1978b. Studies in the Lentibulariaceae 10. The development of gametophytes in Utricularia cornuta Mixch. Plant Science (Lucknow) 8:11-15.

Sohma K. 1975a. Pollen morphology of the Japanese species of Utricularia L. and Pinguicula L. with notes on fossil pollen of Utricularia from Japan (1). Japanese Journal of Botany 50:164-179.

Sohma K. 1975b. Pollen morphology of the Japanese species of Utricularia L. and Pinguicula L. with notes on fossil pollen of Utricularia from Japan (2). Japanese Journal of Botany 50:193-209.

Stolt H. 1936. Beiträge zur Embryologie der Lentibulariaceen. Svensk Botanisk Tidskrift 30:690-696.

Taylor P. 1989. The genus Utricularia. Kew Bulletin 14:1-72.

Taylor P. 1991. The genus Genlisea. Carnivorous Plant Newsletter 20:20-26.

Thanikaimoni G. 1966. Pollen morphology of the genus Utricularia. Pollen et Spores 8:265-284.

Wydler H. 1857. Morphologische Mitteilungen. Flora 40:539.

Zamudio S. 1990. El género Pinguicula (Lentibulariaceae) en México. Resúmenes del VI Congreso Latinoamericano de Botánica, La Habana, Cuba.

Zamudio S. 2001. Revisión de la sección Orcheosanthus, del género Pinguicula (Lentibulariaceae). Tesis doctoral, Universidad Nacional Autónoma de México, Facultad de Ciencias, México, D.F., 225 pp.

Zamudio S. y Ludlow-Wiechers B. 1993. No 4. Lentibulariaceae, Pinguicula. En: Ludlow-Weichers B., Diego-Pérez N. y Márquez-Guzmán J. Eds. Flora Palinológica de Guerrero, Facultad de Ciencias, Universidad Nacional Autónoma de México, México, D.F. 16 pp.

Zamudio S. y Lux A. 1992. Una nueva especie gipsícola de Pinguicula (Lentibulariaceae) de Nuevo León, México. Acta Botanica Mexicana 20:39-44.

Fecha de recepción: 14 de julio de 2004

Versión corregida: 4 de febrero de 2005

Aceptado: 5 de febrero de 2005 